\title{
ALMA detection of the dusty object silhouetted against the S0 galaxy NGC 3269 in the Antlia cluster ${ }^{\star}$
}

\author{
L. K. Haikala ${ }^{1}$, R. Salinas ${ }^{2}$, T. Richtler ${ }^{3}$, M. Gómez ${ }^{4}$, G. F. Gahm ${ }^{5}$, and K. Mattila ${ }^{6}$ \\ 1 Instituto de Astronomía y Ciencias Planetarias de Atacama, Universidad de Atacama, Copayapu 485, Copiapo, Chile \\ e-mail: lauri.haikala@uda.cl \\ 2 Gemini Observatory/NSF's NOIRLab, Casilla 603, La Serena, Chile \\ 3 Departamento de Astronomía, Universidad de Concepción, Concepción, Chile \\ 4 Departamento de Ciencias Físicas, Facultad de Ciencias Exactas, Universidad Andres Bello, Fernández Concha 700, \\ Las Condes 7591538, Chile \\ 5 Stockholm Observatory, AlbaNova University Centre, Stockholm University, 10691 Stockholm, Sweden \\ ${ }^{6}$ Department of Physics, University of Helsinki, PO Box 64, 00014 Helsinki, Finland
}

Received 21 July 2020 / Accepted 30 October 2020

\begin{abstract}
Context. An intriguing silhouette of a small dust patch can be seen against the disk of the S0 galaxy NGC 3269 in the Antlia cluster in optical images. The images do not provide any clue as to whether the patch is a local Jupiter mass-scale cloudlet or a large extragalactic dust complex.

Aims. We aim to resolve the nature of this object: is it a small Galactic cloudlet or an extragalactic dust complex?

Methods. ALMA and APEX spectroscopy and Gemini GMOS long-slit spectroscopy were used to measure the velocity of the patch and the NGC 3269 disk radial velocity curve.

Results. A weak $16 \pm 2.5 \mathrm{~km} \mathrm{~s}^{-1}$ wide ${ }^{12} \mathrm{CO}(2-1) T_{\mathrm{MB}} 19 \pm 2.5$. $\mathrm{mK}$ line in a $2^{\prime \prime} .2$ by $2^{\prime \prime} .12$ beam associated with the object was detected with ALMA. The observed heliocentric velocity, $V_{r \text {,hel }}=3878 \pm 5.0 \mathrm{~km} \mathrm{~s}^{-1}$, immediately establishes the extragalactic nature of the object. The patch velocity is consistent with the velocity of the nucleus of NGC 3269, but not with the radial velocity of the NGC 3269 disk of the galaxy at its position. The $\sim 4^{\prime \prime}$ angular size of the patch corresponds to a linear size of $\sim 1 \mathrm{kpc}$ at the galaxy's Hubble distance of $50.7 \mathrm{Mpc}$. The mass estimated from the ${ }^{12} \mathrm{CO}(2-1)$ emission is $\sim 1.4 \times 10^{6}(d / 50.7 \mathrm{Mpc})^{2} M_{\odot}$, while the attenuation derived from the optical spectrum implies a dust mass of $\sim 2.6 \times 10^{4}(d / 50.7 \mathrm{Mpc})^{2} M_{\odot}$. The derived attenuation ratio $A_{B}^{\prime} /\left(A_{B}^{\prime}-A_{R}^{\prime}\right)$ of $1.6 \pm 0.11$ is substantially lower than the corresponding value for the mean Milky Way extinction curve for point sources (2.3). Conclusions. We established the extragalactic nature of the patch, but its origin remains elusive. One possibility is that the dust patch is left over from the removal of interstellar matter in NGC 3269 through the interaction with its neighbour, NGC 3268.
\end{abstract}

Key words. galaxies: individual: 3269 - galaxies: ISM - dust, extinction

\section{Introduction}

The projection of a Galactic dust feature onto a distant galaxy is very rare. To our knowledge, only Dirsch et al. (2003, 2005) explicitly consider such a case. They noted a tiny dust patch with a diameter of about 4" (hereafter referred to as "the patch") and two nearby smaller 0".5 diameter patches seen as silhouettes against the S0 galaxy NGC 3269 located in the area of the Antlia cluster. Dirsch et al. (2005) estimated the B-band attenuation caused by the patch to be approximately one magnitude. A recent high-quality Sloan $r$-band image, taken with Inamori-Magellan Areal Camera \& Spectrograph (IMACS) at the Magellan Baade Telescope, shows the structure of the patch more clearly than before (Fig. 1). The smoothed and contrast-enhanced inset in Fig. 1 reveals that the patch consists of three arcsecond-scale attenuation maxima surrounded by a less dense cometary-shaped halo. The optical images provide only an upper limit for the distance of the patch, that of NGC 3269, and thus the linear size and nature of the object is unknown.

* Molecular line spectra (spectra extracted from a spatially smoothed ALMA image as a FITS binary table) are only available at the CDS via anonymous ftp to cdsarc.u-strasbg. fr $(130.79 .128 .5)$ or via http://cdsarc.u-strasbg.fr/viz-bin/cat/J/A+A/645/A36
Sub-arcsecond to $20^{\prime \prime}$ dusty objects, such as globulettes, residing in $\mathrm{H}$ II regions are detected optically (e.g., Reipurth et al. 2003; De Marco et al. 2006; Gahm et al. 2007) and even in the NIR (e.g. Mäkelä et al. 2014), as they are seen as silhouettes against the bright nebular background emission. Despite their small sizes $\left(4^{\prime \prime}-20^{\prime \prime}\right)$, the globulettes in the Rosette and Carina nebulae were well detected in ${ }^{12} \mathrm{CO}$ and ${ }^{13} \mathrm{CO}(3-2)$ and $(2-1)$ lines at Atacama pathfinder experiment (APEX) (Gahm et al. 2013; Haikala et al. 2017). It has been suggested (Lawrence 2001) that some of the common unidentified faint sub-millimetre sources found in recent surveys are small cold $(7 \mathrm{~K})$ clouds of dust and gas with masses of $\sim 10^{-4}-10^{-2} M_{\odot}(0.1-10$ Jupiter masses) residing in our neighbourhood, $r<100 \mathrm{pc}$. In a blind single-dish $\mathrm{CO}(1-0)$ emission-line search, followed up by high-angular resolution $\left(\sim 3^{\prime \prime}\right)$ interferometry, Heithausen (2002, 2004, 2006) detected cloudlets with sizes of a few hundred AU if at the adopted distance of $100 \mathrm{pc}$, suggesting that such clumpuscules may be an abundant phenomenon in the local interstellar space. Detecting the clumpuscules optically is practically impossible, because if they are not associated with H II regions, they lack bright background emission. Even if detected, the optical images would not provide any clues on the distance to clumpuscules unless they were associated with a known source. 


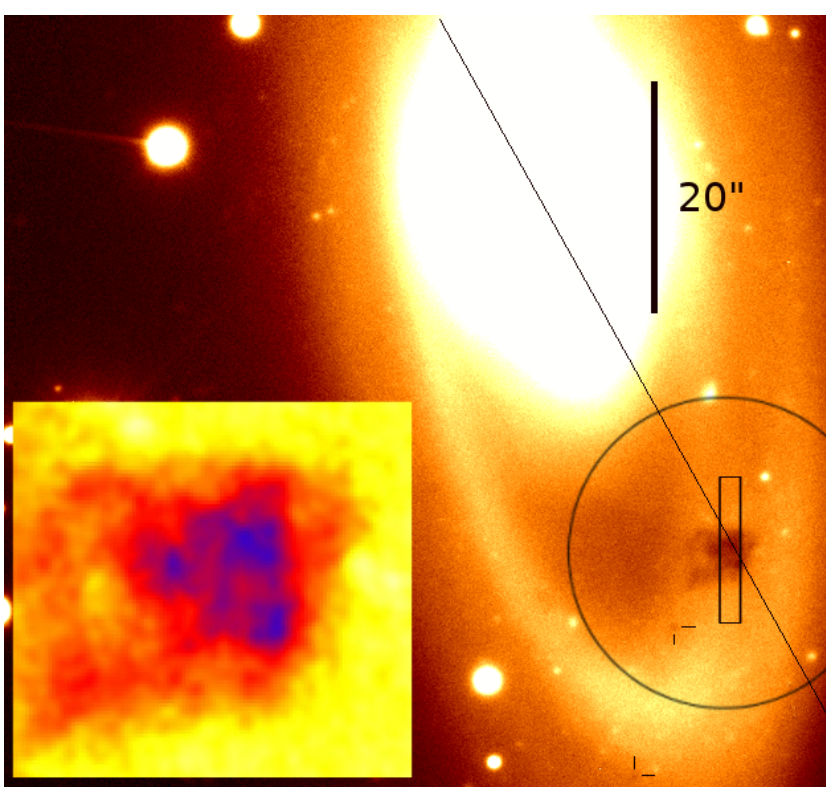

Fig. 1. Detail of Magellan telescope IMACS Sloan $r$-band image of NGC 3269. North is up and east to the left. The patch lies 34" southwest of the bright nucleus. The line indicates the orientation of the GMOS long-slit through the nucleus and the patch. The two tiny patches and the virtual slit used in Dirsch et al. (2005) are indicated. The circle shows the full width at half maximum (FWHM) of the ALMA primary beam at $228 \mathrm{GHz}$. The $6^{\prime \prime}$ by $6^{\prime \prime}$ inset in the lower left shows a smoothed contrast-enhanced Magellan Instant Camera (MagIC) $B$-band image (Dirsch et al. 2005) of the patch.

The patch could be a tiny, nearby $400 \mathrm{AU}$ diameter subJupiter mass clumpuscule in the Milky Way at a distance of $\sim 100$ pc. This would be the first optical detection of a clumpuscule. The association with NGC 3269 would also be intriguing. NGC 3269 is a S0 galaxy with a grand design spiral pattern that is still visible (Fig. 1), and with a heliocentric radial velocity of $3750-3797 \mathrm{~km} \mathrm{~s}^{-1}$. There are no signs of actual or recent star formation. The existence of an isolated dust complex far from the centre is also very atypical for the appearance of dust in earlytype galaxies. Moreover, the galaxy seems to be devoid of $\mathrm{H} \mathrm{I}$ (Barnes \& Webster 2001), with an upper limit of about $10^{9} M_{\odot}$. If associated with NGC 3269, the patch would be an extragalactic giant gas/dust cloud complex larger than $500 \mathrm{pc}$ in diameter.

We utilised the APEX telescope and followed up with the ALMA $12 \mathrm{~m}$ array to search for CO emission in the direction of the patch. Detection of $\mathrm{CO}$ emission from the patch at Galactic velocities around zero would provide the first freely floating sub-Jupiter mass object that can be investigated in detail both in radio and optical. On the other hand, a radial velocity near that of NGC 3269 would confirm its extragalactic nature. In addition, we obtained an optical spectrum with the Gemini MultiObject Spectrograph (GMOS) at Gemini South to measure the radial velocity of the NGC 3269 disk at the position of the patch. Observations and data reduction are described in Sect. 2. The results are presented in Sect. 3 and discussed in Sect. 4, and the conclusions are summarised in Sect. 5.

\section{Observations and data reduction}

\subsection{APEX}

Observations (project O-079F-9321A, PI Gahm) of the ${ }^{12} \mathrm{CO} J=$ (3-2) transition at $345.796 \mathrm{GHz}$ were carried out on
Table 1. ALMA Cycle $5{ }^{12} \mathrm{CO}(2-1)$ observations.

\begin{tabular}{lccccc}
\hline \hline Band & $\begin{array}{c}v_{\text {cen }} \\
{[\mathrm{GHz}]}\end{array}$ & $\begin{array}{c}\text { Width } \\
{[\mathrm{MHz}]}\end{array}$ & $\begin{array}{c}\Delta v \\
{[\mathrm{MHz}]}\end{array}$ & $\begin{array}{c}\Delta v \\
{\left[\mathrm{~km} \mathrm{~s}^{-1}\right]}\end{array}$ & $\begin{array}{c}\mathrm{rms} \\
{[\mathrm{mK}]}\end{array}$ \\
\hline Galactic & 230.414 & 468.8 & 0.244 & 0.318 & 2.0 \\
Intergalactic & 229.726 & 938 & 0.488 & 0.635 & 1.3 \\
Extragalactic & 228.327 & 1875 & 0.977 & 1.28 & 1.0 \\
\hline
\end{tabular}

September 27, 2007 in good weather (PWV $0.34 \mathrm{~mm}$ ) with the $12 \mathrm{~m}$ APEX telescope at Llano Chajnantor, Chile. We used the double-side-band (DSB) heterodyne SIS-receiver APEX-2A mounted on the APEX Nasmyth-A focus. All observations were performed in position-switching mode. The telescope FWHM is $18^{\prime \prime}$ at $345 \mathrm{GHz}$, and the main-beam efficiency is 0.73 . The pointing is estimated to be within $2^{\prime \prime}$. The FFTS1 spectrometer had a bandwidth of $1 \mathrm{GHz}$ and a channel width of $0.4883 \mathrm{MHz}$, corresponding to a channel width of $0.423 \mathrm{~km} \mathrm{~s}^{-1}$ at $345 \mathrm{GHz}$. Calibration was achieved by the chopper wheel method. The difference in the atmospheric opacities in the two side-bands was estimated using an atmospheric model and was taken into account in the calibration. Typical values for the effective DSB system temperatures outside the atmosphere were around $170 \mathrm{~K}$.

Two spectra, each covering $\sim 870 \mathrm{~km} \mathrm{~s}^{-1}$ in velocity, one centred at $0 \mathrm{~km} \mathrm{~s}^{-1}$ and another at $3799 \mathrm{~km} \mathrm{~s}^{-1}$, were obtained. The total on-source integration times were $12 \mathrm{~min}$ and $51 \mathrm{~min}$, respectively. The first-order baseline was subtracted, and the resulting spectrum $\mathrm{rms}$ in $T_{\mathrm{A}}^{*}$ scale was $0.04 \mathrm{~K}(0.029 \mathrm{~K}$ after Hanning-smooth) for the spectrum centred at $0.0 \mathrm{~km} \mathrm{~s}^{-1}$. For the second spectrum, the rms was $0.02 \mathrm{~K}(0.015 \mathrm{~K})$.

\subsection{ALMA $12 \mathrm{~m}$}

The patch was observed during ALMA Cycle 5 (Project 2017.1.00066.S) in the band- $6{ }^{12} \mathrm{CO}(2-1)$ line at $230.538 \mathrm{GHz}$. Three spectral bands covered all the velocities from $-150 \mathrm{~km} \mathrm{~s}^{-1}$ up to $4075 \mathrm{~km} \mathrm{~s}^{-1}$, and one band was dedicated to continuum. Details of the observations are given in Table 1. The observations were conducted in good weather conditions (PWV $0.677 \mathrm{~mm}$ or $2.75 \%$ ) on August 16, 2018 using 44 antennas in configuration $\mathrm{C} 43-2$ (baselines $15 \mathrm{~m}$ to $500 \mathrm{~m}$, maximum resolvable scale $\left.7{ }^{\prime \prime} 8\right)$. The field was centred at $10^{\mathrm{h}} 29^{\mathrm{m}} 55^{\prime \prime} .8,-35^{\circ} 13^{\prime} 58^{\prime \prime} 0$ $\mathrm{J} 2000.0$, and the achieved spatial resolution was $00^{\prime \prime} .73$ by $0^{\prime \prime} .96$, position angle $-80^{\circ}$.

\subsection{Gemini-S spectroscopy}

The galaxy NGC 3269 was observed with the Gemini South telescope, located in Cerro Pachón, Chile, using GMOS (Hook et al. 2004), on the nights of February 4 and 5, 2019, under programme GS-2018B-FT-208. GMOS is equipped with three Hamamatsu CCDs (Gimeno et al. 2016), for a field-of-view of $5.5^{\prime} \times 5.5^{\prime}$. A $1^{\prime \prime}$ long-slit together with the B600 grating gave a $4.6 \AA$ spectral resolution. The slit was positioned and aligned to include both the galaxy's optical centre and the patch. In total, ten exposures of $1200 \mathrm{~s}$ each were obtained, together with spectroscopic flats and $\mathrm{CuAr}$ arcs obtained at similar elevation.

Data reduction was conducted with standard techniques implemented within the Gemini IRAF package. The wavelength zero point was corrected by measuring the position of the bright sky line O I $6300.30 \AA$ after bias subtraction, flat-fielding and wavelength calibration. Two-dimensional spectra were then 


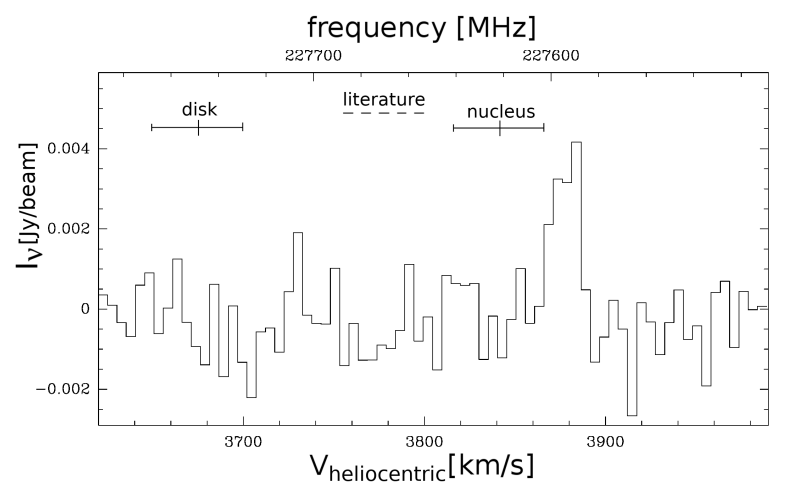

Fig. 2. Twice-Hanning-smoothed ALMA ${ }^{12} \mathrm{CO}(2-1)$ spectrum in a $2^{\prime \prime 2}$ by 2 ". 12 beam towards the patch. The heliocentric radial velocity of the NGC3269 disk and nucleus as measured from the GMOS spectrum and the range of NGC3269 velocity values in the literature are indicated.

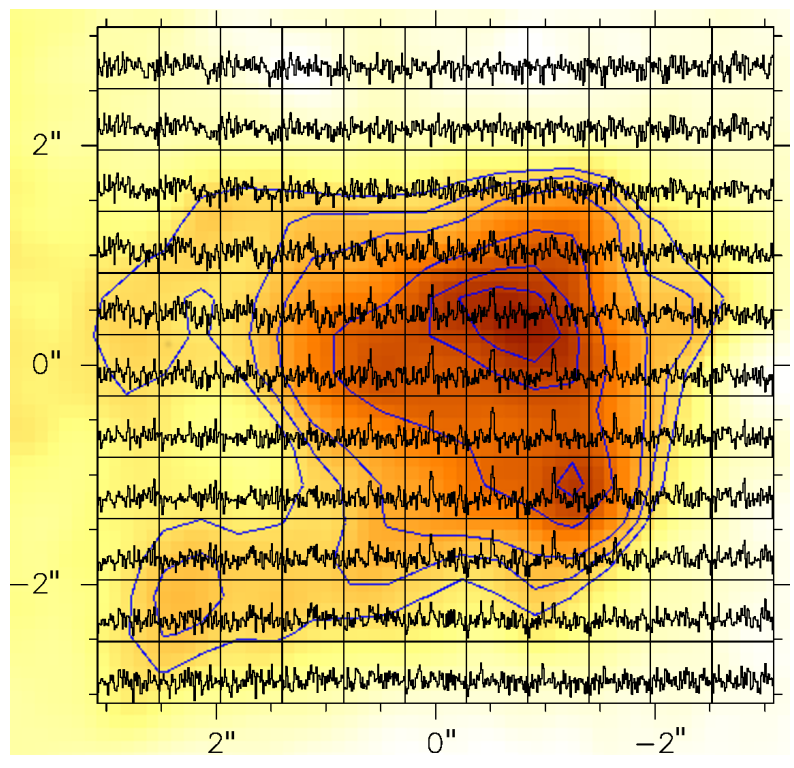

Fig. 3. Smoothed Magellan telescope IMACS $g$-band image of the patch. The contours at arbitrary levels follow the drop in the relative optical surface brightness. Overlaid are ALMA ${ }^{12} \mathrm{CO}(2-1)$ spectra smoothed by a $2^{\prime \prime}$ Gaussian in the direction of the patch. The spectra are twice Hanning-smoothed.

combined with the task gemscombine, and sky was subtracted with a sky sample $\sim 75^{\prime \prime}$ away from the galaxy centre, where no galaxy signal is visible. Spectra were finally extracted at different radii with apall.

\subsection{Magellan IMACS imaging}

The galaxy NGC 3269 was observed with the Magellan IMACS $\mathrm{f} / 48 \mathrm{Kx} 8 \mathrm{~K}$ CCD mosaic camera (pixel size $0{ }^{\prime \prime} .11$ ) on the night of February 10, 2018. $3 \times 600 \mathrm{~s}, 2 \times 600 \mathrm{~s}$ and $2 \times 600 \mathrm{~s}$ exposures were taken in Sloan $g, r$, and $i$, respectively, with an average seeing of $0.45^{\prime \prime}$. Images were reduced with THELI (Schirmer 2013), including bias subtraction, flat-fielding, mosaicing of the eight CCDs, and final co-addition.

\section{Results}

No line was detected above two sigma levels in either of the spectral windows observed at APEX. The patch was therefore

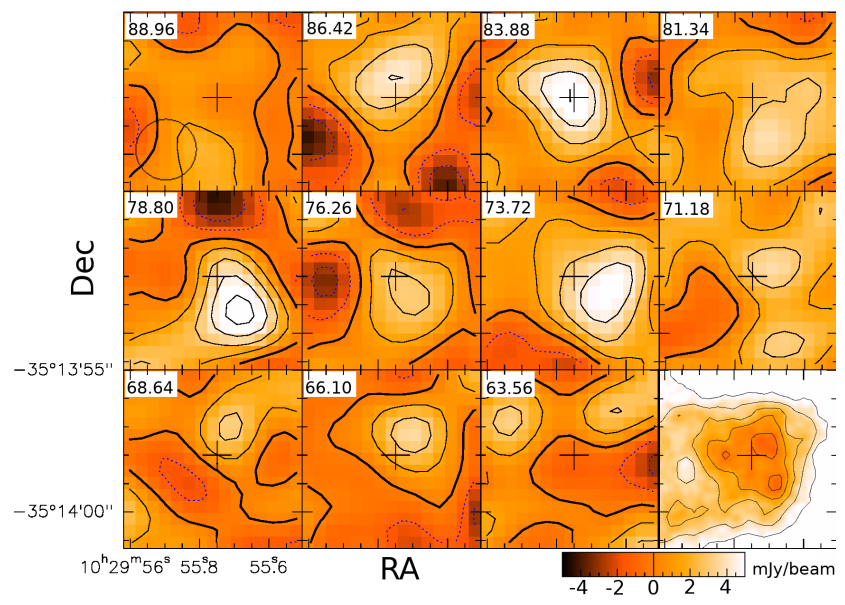

Fig. 4. Channel map of once-Hanning-smoothed spectra in the direction of the patch. The velocity $V_{r \text {,hel }}-3800 \mathrm{~km} \mathrm{~s}^{-1}$ is shown in the upperleft corner of each panel. The cross marks the ALMA field phase centre, and the ALMA smoothed 2'.2 by 2 ".12 beam is shown in the panel in the upper-left. The contours are from $0 \mathrm{mJy} \mathrm{beam}^{-1}$ (thick contour) in one $\sigma$ step of a $1.4 \mathrm{mJy}_{\text {beam }}^{-1}$. Magellan Clay telescope MagIC $B$ band image of the patch is shown in the lower-right, where the contours at arbitrary levels follow the drop in the relative optical surface brightness.

re-observed with ALMA, this time also including the intergalactic velocities. The three ALMA images were smoothed to a 2 ".2 by 2 ". 12 beam and twice Hanning-smoothed to a channel width of $5.13 \mathrm{~km} \mathrm{~s}^{-1}$ in velocity to improve the noise level. No signal above the noise was detected in the ALMA images centred on Galactic and intergalactic velocities. A weak line was detected in the direction of the patch in the spectral window centred on extragalactic velocities. The line intensity and line width from a Gaussian fit to the spectrum are $3.8 \mathrm{mJy}_{\text {beam }}{ }^{-1} \pm 0.5 \mathrm{mJy}_{\text {beam }}{ }^{-1}\left(19 \mathrm{mK} \pm 2.5 \mathrm{mK}\right.$ in $T_{\mathrm{MB}}$ scale) and $16.0 \mathrm{~km} \mathrm{~s}^{-1} \pm 2.3 \mathrm{~km} \mathrm{~s}^{-1}$, respectively. The LSR centre of line velocity is $3827 \mathrm{~km} \mathrm{~s}^{-1} \pm 5.0 \mathrm{~km} \mathrm{~s}^{-1}$, which, when expressed according to the optical redshift convention, corresponds to a heliocentric velocity of $3878 \mathrm{~km} \mathrm{~s}^{-1}$ (Fig. 2).

As a consistency check, we estimated the expected APEX $\mathrm{CO}(3-2)$ line strength based on our ALMA CO (2-1) detection. The dominating effect comes from the beam dilution: for a $4^{\prime \prime}$ Gaussian distribution of the patch intensity, the dilution factor in an $18^{\prime \prime}$ beam is $\sim 20$. Adopting a $\mathrm{CO}(3-2)$ to $\mathrm{CO}(2-1)$ line intensity ratio of $\sim 0.6$ (Lamperti et al. 2020), the expected antenna temperature of the $\mathrm{CO}(3-2)$ line is $\sim 19 \mathrm{mK} / 33$ or $\sim 0.6 \mathrm{mK}$. Compared with the $15 \mathrm{mK} \mathrm{rms}$, this means that the sensitivity of our APEX observation was far below the detection limit required for the patch.

Twice-Hanning-smoothed spectra in the direction of the patch are shown in Fig. 3 overlaid on the smoothed Magellan telescope IMACS Sloan $g$-band image. The contours trace the drop in relative optical surface brightness of the patch. The image confirms that the line emission originates in the patch and that no other line above the noise is seen outside the patch.

A channel map of ALMA spectra once Hanning-smoothed to channel width $2.5 \mathrm{~km} \mathrm{~s}^{-1}$ in the direction of the patch is shown in Fig. 4. The optical attenuation in the field is shown in the lower-right. The two stronger attenuation maxima seen in the inset in Fig. 1 can be resolved in the channel map. The weaker easternmost maximum is not seen, possibly due to the heavy spatial smoothing. 


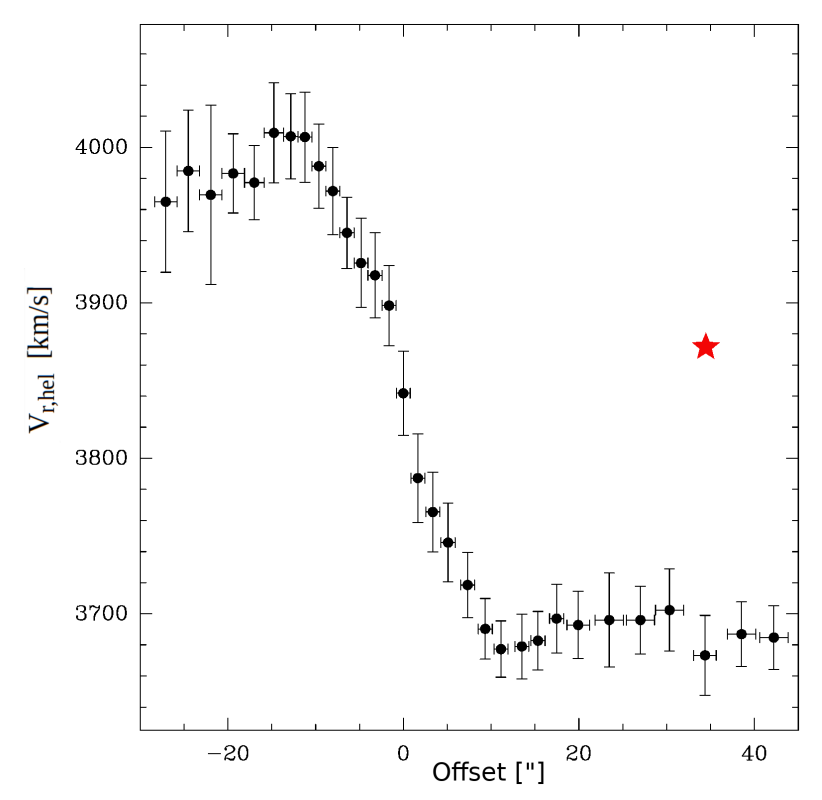

Fig. 5. Heliocentric radial velocities as measured from the GMOS longslit spectrum. The $x$-axis is the distance in arc seconds along the slit from the galaxy nucleus. The patch is at an offset of $34^{\prime \prime}$. The velocity of the patch is shown with a red star.

The rms of the ALMA continuum image in the direction of the patch is $20 \times 10^{-6} \mathrm{Jy}$ in a $1^{\prime \prime}$ beam. No sources above the noise are detected in the image.

The NGC 3269 radial velocities along the GMOSS slit were measured from the extracted spectra with the crosscorrelation procedure (Tonry \& Davis 1979) as implemented in IRAF/fxcor. The spectra were cross-correlated with 28 single stellar population synthesis models from Vazdekis et al. (2016), with a $1-14$ Gyr range of ages and $-1.3<[\mathrm{M} / \mathrm{H}]<0.2$ dex metallicity. The five results with the highest cross-correlation peaks were averaged for a final velocity per radial bin. The best fit SSPs were old $(10-14 \mathrm{Gyr})$ and super solar $([\mathrm{M} / \mathrm{H}]=0.2)$ for the centre of the galaxy, and intermediate age ( $2 \mathrm{Gyr})$ and solar $([\mathrm{M} / \mathrm{H}]=0.0)$ for the disk. The resulting radial velocities along the slit are shown in Fig. 5.

\section{Discussion}

\subsection{Radial velocity of the patch}

The observed CO line velocity, $V_{r \text {,hel }}=3878 \pm 5.0 \mathrm{~km} \mathrm{~s}^{-1}$, agrees within the error limits with the radial velocity of the NGC 3269 nucleus, $V_{r \text {,hel }}=3840 \pm 26 \mathrm{~km} \mathrm{~s}^{-1}$ as measured from the GMOS spectrum. This confirms the extragalactic nature of the patch and also shows that it is likely to be at the same distance as and that associated with NGC 3269. The radial velocity of the NGC 3269 disk in the direction of the patch has a radial velocity of $V_{r \text {,hel }}=3673 \pm 25 \mathrm{~km} \mathrm{~s}^{-1}$, that is, $\sim 200 \mathrm{~km} \mathrm{~s}^{-1}$ smaller than that of the patch. This excludes the possibility that the patch could be a molecular cloud complex embedded in or participating alongside the rotation of the galactic disk. Depending on its tangential velocity component, the patch could be falling into, orbiting around, or passing by the galaxy NGC 3269.

At the Hubble distance of NGC 3269, 50.7 Mpc, the 4" diameter of the patch corresponds to nearly $1 \mathrm{kpc}$. The $\sim 1^{\prime \prime}$ size attenuation maxima seen in Fig. 1 inset have extensions of $\sim 200 \mathrm{pc}$ each; the patch is thus a large molecular cloud complex.
Given the substantial maximum optical attenuation of $A_{B}^{\prime} \sim 1 \mathrm{~m} 0$ in the patch (Dirsch et al. 2005), the low intensity of the observed ${ }^{12} \mathrm{CO}(2-1)$ line of $\sim 0.3 \mathrm{~K} \mathrm{~km} \mathrm{~s}^{-1}$ as compared to $\gtrsim 10$ times larger values in Galactic molecular clouds with similar extinction, is striking. Possible explanations are that either the metallicity or the beam filling of the molecular gas component in the patch is extremely low. Even below the $\sim 1^{\prime \prime}$ clumping, seen in the optical image (Fig. 1, inset), the patch could consist of a large number of clouds with apparent diameters of only a fraction of an arcsecond, explaining the small beam filling.

\subsection{Mass of the patch}

The mass of the patch can be estimated from the ${ }^{12} \mathrm{CO}(2-1)$ emission. The average CO line integral $W_{\mathrm{CO}}=\int T \mathrm{~d} V$ over velocities $3863 \mathrm{~km} \mathrm{~s}^{-1}$ to $3894 \mathrm{~km} \mathrm{~s}^{-1}$ in an $22.6 \operatorname{arcsec}^{2}$ area where the line is detected (inside offsets -2 '.5 to $2^{\prime \prime} .5$ in RA and -2 ".5 to 2 ".0 in Dec in Fig. 3), is $0.92 \mathrm{~K} \mathrm{~km} \mathrm{~s}^{-1} \operatorname{arcsec}^{-2}$. Adopting the Herrera et al. (2020) conversion factor $\alpha_{\mathrm{CO}}(2-1)$ of $6.2 M_{\odot} \mathrm{pc}^{-2}\left(\mathrm{~K} \mathrm{~km} \mathrm{~s}^{-1}\right)^{-1}$, we obtain the patch mass of $(\mathrm{d} / 50.7 \mathrm{Mpc})^{2} \times 1.4 \times 10^{6} M_{\odot}$, where $\mathrm{d}$ is the true distance to NGC 3269.

\subsection{Mass of the dust}

The mass of dust in the patch can be estimated by using the attenuation ${ }^{1}$ of the background light passing through the patch. The GMOS spectrum of the surface brightness distribution across the patch was used to measure the attenuation over the wavelength range of $\sim 4000 \AA-6800 \AA$. The spectrum was re-binned to 0 ".64 in spatial coordinate along the slit, and the attenuation was estimated by adopting a background intensity level 4 " southwest of the patch. The NGC 3269 disk surface brightness in this position is similar to the background intensity in the Dirsch et al. (2005) virtual slit. It is assumed that the spectral energy distribution in the off position is the same as that behind the patch. The detector gaps, bad pixels, and (because of the low $\mathrm{S} / \mathrm{N}$ ) the wavelengths below $4000 \AA$ were masked. The resulting wavelength-dependent attenuation, after re-binning by 20 in wavelength, is shown in Fig. 6 for a $00^{\prime \prime} 64$ broad slice at the position of the maximum attenuation. The blue line is a thirddegree polynomial fit to the data. The red and the dashed green lines show the extinction wavelength dependence according to Cardelli et al. (1989) corresponding to $A_{v} / E(B-V)$ values of 3.1 and 1.5 . Our attenuation curve is seen to closely follow the latter extinction curve.

Dirsch et al. (2005) estimated the maximum and the mean $B$-band attenuation in a $4^{\prime \prime}$ diameter area in the patch to be $A_{B}^{\prime} \sim 1 \mathrm{~m} .0$ and 0.4 , respectively. Their maximum attenuation is somewhat higher than the value $A_{B}^{\prime} \approx 0.8 \pm 0.05$ obtained from the GMOS spectrum. This difference is possibly due to the positioning of the GMOS spectrum slit that does not pass exactly through the densest part of the patch, and also to the selected off position. Had the off position been chosen northeast of the patch, the attenuation would be $10 \%$ lower, but the wavelength dependence of the attenuation would not change. Our $A_{B}^{\prime} /\left(A_{B}^{\prime}-A_{R}^{\prime}\right)$

1 We emphasise the difference between the concepts of extinction for point sources and attenuation as obtained from dimming of background surface brightness that passes through a foreground dust screen. The intensity ratio between the patch and the background gives the attenuation, which in magnitudes is expressed as $A^{\prime}=$ $-2.5 \log (I$ (patch $) / I$ (background $))$. 


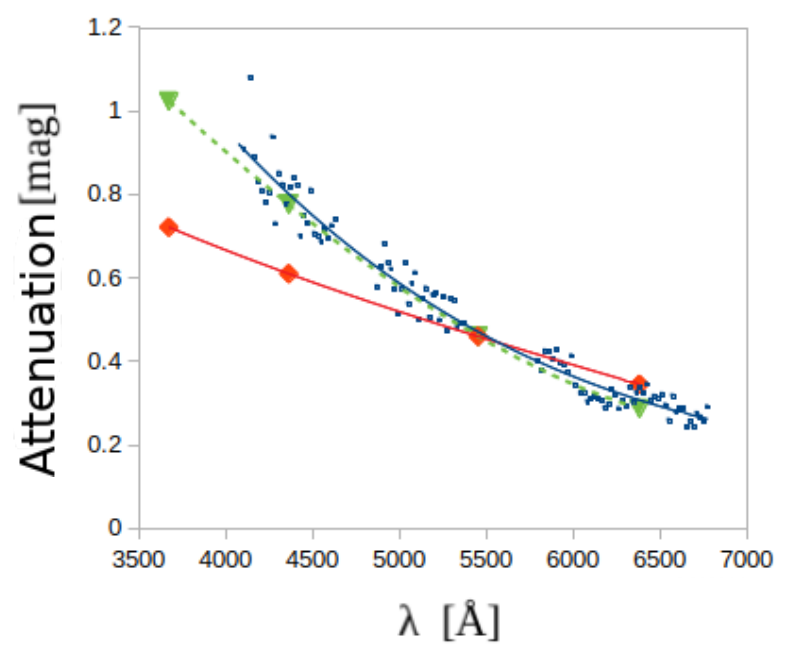

Fig. 6. Attenuation as calculated for a 0'.64 broad slice of GMOS spectrum in the position of the maximum attenuation of the patch. GMOS pixels have been binned by 20 in the wavelength. The blue line is a fit to the data. Extinction according to Cardelli et al. (1989) is shown for $A_{v} / E(B-V)$ values of 1.5 (green triangles, dashed line) and 3.1 (red diamonds, continuous line), respectively.

ratio $(1.6 \pm 0.11)$ is the same as $(1.6 \pm 0.25)$ reported in Dirsch et al. (2005) and it is, again, substantially lower than the corresponding value for the mean Milky Way extinction curve for point sources, $A_{B} /\left(A_{B}-A_{R}\right)=2.3$.

The background surface brightness attenuation in a clumpy foreground screen has been modelled by Natta \& Panagia (1984). Clumping causes the reddening curve to flatten, meaning $R_{V}^{\prime}$ and $A_{B}^{\prime} /\left(A_{B}^{\prime}-A_{R}^{\prime}\right)$ increase as compared to a homogeneous screen. Thus, clumping cannot explain the steeper wavelength dependence as observed for the patch. In several cases, dust lanes or patches have been observed in early-type galaxies with $R_{V}^{\prime}$ values as low as 1.9 (IC 4320, Warren-Smith \& Berry 1983) and 2.15 (NGC 5626, Goudfrooij et al. 1994). Such cases have been interpreted as evidence for dust-grain size distributions with mean and maximum grain sizes smaller than those for the mean Milky Way extinction curve (with $R_{V}=3.1$ ).

The dust mass for lanes and patches in galaxies has frequently been estimated using the following formula:

$M_{d}=\Sigma\left\langle A_{B}^{\prime}\right\rangle \Gamma_{B}^{-1}$,

where $\left\langle A_{B}^{\prime}\right\rangle$ is the mean attenuation as measured over the object's area of $\Sigma$ (Sadler \& Gerhard 1985; van Dokkum \& Franx 1995). For the Milky Way's mean dust properties, the value of the mass absorption coefficient $\Gamma_{B}$ is $\Gamma=4 \times 10^{4} \mathrm{mag} \mathrm{cm}^{2} \mathrm{~g}^{-1} \sim 8 \times$ $10^{-6}$ mag kpc ${ }^{2} M_{\odot}^{-1}$. Adopting $\left\langle A_{B}^{\prime}\right\rangle=0^{\mathrm{m}} .4$ as the mean attenuation over the area of $\Sigma=4^{\prime \prime} \times 4^{\prime \prime}$ of the patch, we obtain the following for its dust mass: $M_{d}=4.8 \times 10^{4}(d / 50.7 \mathrm{Mpc})^{2} M_{\odot}$.

Goudfrooij et al. (1994) presented a method to estimate the value of $\Gamma_{B}$ for extinction (attenuation) curves with $R_{V}^{\prime}$ values other than 3.1. For NGC 5626 and IC 4320, which have the smallest values of $R_{V}^{\prime}$ in their sample (2.15 and 2.08), they found that the dust masses as obtained from the above formula have to be multiplied by a factor of $\sim 0.66$. In the case of the patch with $R_{V}^{\prime}=1.5$, we estimate that a correction factor of $\sim 0.5$ could be appropriate. Thus the dust mass estimate for the patch becomes $M_{d} \sim 2.6 \times 10^{4}(d / 50.7 \mathrm{Mpc})^{2} M_{\odot}$, which corresponds to a molecular-gas-to-dust ratio of $\sim 55$. If at the distance of $24.3 \mathrm{Mpc}$ as implied by the Tully-Fisher argument (see Sect. 4.4), the mass of the dust and molecular gas would be $\sim 6 \times 10^{3} M_{\odot}$ and $\sim 0.3 \times 10^{6} M_{\odot}$, respectively, and the moleculargas-to-dust ratio would not change.

The attenuation of the patch caused by the dust is modest and it is very probable that the patch also contains a large amount of atomic hydrogen. With an amount similar to the molecular hydrogen, the gas-to-dust mass would be close to the Milky Way standard value of 100-150. The NGC 3269 atomic hydrogen mass upper limit for an assumed $200 \mathrm{~km} \mathrm{~s}^{-1}$ line width is $\sim 10^{8} M_{\odot}$ (Barnes \& Webster 2001). Even for a much narrower line, the H I mass upper limit remains much above the molecular gas mass of the patch and the HI gas could thus easily respond to the need to make the gas-to-dust mass similar to the Milky Way standard value.

In a surface brightness distribution like the face of a galaxy, instrumental and atmospheric "PSF blurring" tends to fill in small size $\left(1^{\prime \prime}-4\right.$ ") depressions or "holes", such as the patch and its sub-structures. Light from the surrounding region of higher surface brightness is being poured into "the dark hole". Given the instrumental + atmospheric PSF this effect could, at least approximately, be corrected for as has been discussed by McCaughrean \& O'dell (1996). They analysed the effect in the case of their HST imaging of circumstellar disks, seen silhouetted as dark markings against the bright background of the Orion Nebula. They found that the "PSF blurring" became an important correction for their $\lesssim 0.5^{\prime \prime}$ sized objects. Given the substantially broader core and wider wings of the PSFs of ground-based telescopes, the blurring effect is already important for source structures of $1^{\prime \prime}-4^{\prime \prime}$, as in the patch. Correction of this effect has not been attempted for the patch. It would have had the effect of increasing the attenuation and thereby the dust mass estimate.

\subsection{Origin of the patch}

The confirmation of the patch as a projected Galactic dust cloud would have been an intriguing finding. However, now that its extragalactic nature is obvious, the resulting questions are no less intriguing. Dust in early-type galaxies is frequent, but an isolated large dust patch in a galaxy without young stellar populations is, to our knowledge, very rare (if not unique) in the literature. A morphologically similar patch in NGC 3923 was found by Sikkema et al. (2007). However, NGC 3923 is a prominent shell galaxy with obvious infall of dwarf galaxies, and there is more dust beside that patch. Even in this case, Sikkema et al. (2007) favour an internal origin. This may be the case in NGC 3269 as well. NGC 3269 is a transition object between a grand design spiral and an S0, so isolated dust suggests relation to the quenching of star formation, (i.e. the process of transforming a starforming spiral galaxy into a non-star-forming S0). In that way, one could understand the dust in NGC 3269 as a debris of the former gas/dust component of its disk.

The galaxy NGC 3269 looks tidally undisturbed at first sight. However, the grand spiral design by itself may be a sign of previous tidal influence (e.g. Struck \& Smith 2012; Semczuk et al. 2017). Other traces of a tidal history were noted by Dirsch et al. (2005, see the inset of their Fig. 1), in particular an arc-like structure extending towards the southeast. Because this arc is much more visible in the Washington $C$-filter than in the faster $R$-filter, one suspects that the enhanced brightness is due to the very strong O III-line at $3727 \AA$ in H II-regions that falls into the $C$-filter. It is a clear sign of star formation, but with our present data, we cannot further constrain possible tidal effects.

A viable mechanism of removing gas from galaxies is rampressure stripping by a passage through a hot ambient medium (e.g. Abramson et al. 2011; Jaffé et al. 2018; Cramer et al. 2019). 
The obvious candidate for this process is the extended hot halo of NGC 3268, whose projected distance is only $60 \mathrm{kpc}$, but its radial velocity is $2700 \mathrm{~km} \mathrm{~s}^{-1}, 1000 \mathrm{~km} \mathrm{~s}^{-1}$ less than that of NGC 3269 . By its position, the arc could principally indicate the wake of the passage. Assuming this, NGC 3269 would be in the foreground of NGC 3268, the closest distance yet to come. Otherwise, a passage that close to NGC 3268 would have left a more disturbed structure of both NGC 3268 and NGC 3269. That contradicts the argument of Caso \& Richtler (2015), who put NGC 3269 far in the background. They interpret the radial velocity difference of $1000 \mathrm{~km} \mathrm{~s}^{-1}$ between NGC 3268 and NGC 3269 as a difference of the recession velocities rather than of the Doppler velocities. A further striking feature of that galaxy group around NGC 3268 is that the other two S0-galaxies, NGC 3271 and NGC 3267, show radial velocities of $3804 \mathrm{~km} \mathrm{~s}^{-1}$ and $3709 \mathrm{~km} \mathrm{~s}^{-1}$, respectively, which is almost identical to that of NGC 3269. This group would then be at a distance of about $50 \mathrm{Mpc}$, and tidal interactions between these galaxies would be possible, but ram-pressure effects would lose their plausibility. The Antlia galaxy cluster in this case would be an effect of looking along a filament of galaxies, which results in a superposition of Doppler and recession velocities.

The clear key to this problem lies in the precise individual distances to these three galaxies, which are currently not available. The distance to NGC 3268 is, however, well established.

We can make a rough estimate by assuming that NGC 3269, like all disk galaxies, is on the baryonic Tully-Fisher relation. We adopt the relation $M_{\text {bar }}=50 \times v_{f}^{4}$ (McGaugh 2005) and assume that the observed radial velocities are the circular velocities, which may be wrong, if tidal effects are important. The radial velocity difference between the centre and the constant level in the disk is $150 \mathrm{~km} \mathrm{~s}^{-1}$, the angle of inclination is $27.5^{\circ}$, the position angle of the slit with respect to the major axis is $10^{\circ}$ : this gives $175 \mathrm{~km} \mathrm{~s}^{-1}$ and $M_{\mathrm{bar}}=4.7 \times 10^{10} M_{\odot}$. For an old population, we adopt $M / L=5.0$. In reality, it will be somewhat smaller because of the intermediate-age disk. This gives $9.4 \times 10^{9}$ solar luminosities, or $M_{R}=-20 \mathrm{~m} .7$. The apparent Rmagnitude is $11^{\mathrm{m}} \cdot 73$. With $A_{R}=0 \mathrm{~m} \cdot 2$, one has $m-M=31^{\mathrm{m}} \cdot 9$ or 24.3 Mpc (i.e. in the foreground of NGC 3268 by $10 \mathrm{Mpc}$ ). The uncertainties are unknown and probably large, but a Hubble distance of $50 \mathrm{Mpc}$ is difficult to justify. This raises the problem of explaining the radial velocities of the three S0s. If they are a group, as the very similar radial velocities suggest, the velocity difference to NGC 3268 cannot be due to the gravitational attraction of the Antlia cluster, but must be attributed to a large-scale structure.

\section{Conclusions}

The S0 galaxy NGC 3269 is devoid of gas, but hosts a dust patch, which has previously been suspected as a Galactic tiny dust cloud, projected onto NGC 3269. While APEX observations failed to detect the dust patch in the ${ }^{12} \mathrm{CO}(3-2)$ line, faint ${ }^{12} \mathrm{CO}(2-1)$ emission associated with the dust was detected with ALMA. The patch radial CO velocity, $V_{r \text {,hel }}=3878 \pm$ $5.0 \mathrm{~km} \mathrm{~s}^{-1}$, places it clearly at the galaxy's distance and matches the radial velocity of the galaxy's nucleus. The patch deviates from the radial velocity curve of the disk of NGC 3269, as shown through Gemini GMOSS long-slit spectroscopy. The optical attenuation as estimated from the long-slit spectrum confirms the steepness of the reddening law reported by Dirsch et al. (2005). The gas mass estimate from the CO emission is approximately $1.4 \times 10^{6}(d / 50.7 \mathrm{Mpc})^{2} M_{\odot}$. The origin of the dust patch remains, however, elusive. A plausible speculation is that the gas/dust cloud is debris from the previous gas/dust component of the disk of NGC 3269. A possible process is ram-pressure stripping by the hot halo of the neighbouring galaxy, NGC 3268, which requires NGC 3269 to be in the foreground of NGC 3268. This revives the problem of the nature of the radial velocities of NGC 3269 and its neighbouring S0s, NGC 3267 and NGC 3271: are these recession velocities or Doppler velocities? Robust distances are needed to solve this problem.

Acknowledgements. T.R. acknowledges support from the BASAL Centro de Astrofísica y Tecnologias Afines (CATA) PFB-06/2007. Based partly on observations obtained at the international Gemini Observatory, a program of NSF's NOIRLab, which is managed by the Association of Universities for Research in Astronomy (AURA) under a cooperative agreement with the National Science Foundation. on behalf of the Gemini Observatory partnership: the National Science Foundation (United States), National Research Council (Canada), Agencia Nacional de Investigación y Desarrollo (Chile), Ministerio de Ciencia, Tecnología e Innovación (Argentina), Ministério da Ciência, Tecnologia, Inovações e Comunicações (Brazil), and Korea Astronomy and Space Science Institute (Republic of Korea) and partly on data acquired with the Atacama Pathfinder EXperiment (APEX) (project O-079F-9321A). APEX is a collaboration between the Max-Planck-Institut für Radioastronomie, the European Southern Observatory, and the Onsala Space Observatory. This paper includes data gathered with the 6.5 meter Magellan Telescopes located at Las Campanas Observatory, Chile obtained via CNTAC program CN2018A-83 This paper makes use of the following ALMA data: ADS/JAO.ALMA\#2017.1.00066.S. ALMA is a partnership of ESO (representing its member states), NSF (USA) and NINS (Japan), together with NRC (Canada), MOST and ASIAA (Taiwan), and KASI (Republic of Korea), in cooperation with the Republic of Chile. The Joint ALMA Observatory is operated by ESO, AUI/NRAO and NAOJ.

\section{References}

Abramson, A., Kenney, J. D. P., Crowl, H. H., et al. 2011, AJ, 141, 164 Barnes, D. G., \& Webster, R. L. 2001, MNRAS, 324, 859

Cardelli, J. A., Clayton, G. C., \& Mathis, J. S. 1989, ApJ, 345, 245

Caso, J. P., \& Richtler, T. 2015, A\&A, 584, A125

Cramer, W. J., Kenney, J. D. P., Sun, M., et al. 2019, ApJ, 870, 63

De Marco, O., O’Dell, C. R., Gelfond, P., Rubin, R. H., \& Glover, S. C. O. 2006 , AJ, 131, 2580

Dirsch, B., Richtler, T., \& Bassino, L. P. 2003, A\&A, 408, 929

Dirsch, B., Richtler, T., \& Gómez, M. 2005, AJ, 130, 1141

Gahm, G. F., Grenman, T., Fredriksson, S., \& Kristen, H. 2007, AJ, 133, 1795

Gahm, G. F., Persson, C. M., Mäkelä, M. M., \& Haikala, L. K. 2013, A\&A, 555, A57

Gimeno, G., Roth, K., Chiboucas, K., et al. 2016, in Ground-based and Airborne Instrumentation for Astronomy VI, Proc. SPIE, 9908, 99082S

Goudfrooij, P., de Jong, T., Hansen, L., \& Norgaard-Nielsen, H. U. 1994, MNRAS, 271, 833

Haikala, L. K., Gahm, G. F., Grenman, T., Mäkelä, M. M., \& Persson, C. M. 2017, A\&A, 602, A61

Heithausen, A. 2002, A\&A, 393, L41

Heithausen, A. 2004, ApJ, 606, L13

Heithausen, A. 2006, A\&A, 450, 193

Herrera, C. N., Pety, J., Hughes, A., et al. 2020, A\&A, 634, A121

Hook, I. M., Jørgensen, I., Allington-Smith, J. R., et al. 2004, PASP, 116, 425

Jaffé, Y. L., Poggianti, B. M., Moretti, A., et al. 2018, MNRAS, 476, 4753

Lamperti, I., Saintonge, A., Koss, M., et al. 2020, ApJ, 889, 103

Lawrence, A. 2001, MNRAS, 323, 147

Mäkelä, M. M., Haikala, L. K., \& Gahm, G. F. 2014, A\&A, 567, A108

McCaughrean, M. J., \& O'dell, C. R. 1996, AJ, 111, 1977

McGaugh, S. S. 2005, ApJ, 632, 859

Natta, A., \& Panagia, N. 1984, ApJ, 287, 228

Reipurth, B., Raga, A., \& Heathcote, S. 2003, AJ, 126, 1925

Sadler, E. M., \& Gerhard, O. E. 1985, MNRAS, 214, 177

Schirmer, M. 2013, ApJS, 209, 21

Semczuk, M., Łokas, E. L., \& del Pino, A. 2017, ApJ, 834, 7

Sikkema, G., Carter, D., Peletier, R. F., et al. 2007, A\&A, 467, 1011

Struck, C., \& Smith, B. J. 2012, MNRAS, 422, 2444

Tonry, J., \& Davis, M. 1979, AJ, 84, 1511

van Dokkum, P. G., \& Franx, M. 1995, AJ, 110, 2027

Vazdekis, A., Koleva, M., Ricciardelli, E., Röck, B., \& Falcón-Barroso, J. 2016, MNRAS, 463, 3409

Warren-Smith, R. F., \& Berry, D. S. 1983, MNRAS, 205, 889 